\title{
When Ostrom Meets Blockchain: Exploring the Potentials of Blockchain for Commons Governance
}

\author{
David Rozas' ${ }^{1}$, Antonio Tenorio-Fornés' (D), \\ Silvia Díaz-Molina', and Samer Hassan ${ }^{1,2}$
}

\begin{abstract}
Blockchain technologies have generated enthusiasm, yet their potential to enable new forms of governance remains largely unexplored. Two confronting standpoints dominate the emergent debate around blockchain-based governance: discourses characterized by the presence of techno-determinist and market-driven values, which tend to ignore the complexity of social organization; and critical accounts of such discourses which, while contributing to identifying limitations, consider the role of traditional centralized institutions as inherently necessary to enable democratic forms of governance. In this article, we draw on Ostrom's principles for self-governance of communities to explore the transformative potential of blockchain beyond such standpoints. We approach blockchain through the identification and conceptualization of six affordances that this technology may provide to communities: tokenization, self-enforcement and formalization of rules, autonomous automatization, decentralization of power over the infrastructure, increasing transparency, and codification of trust. For each affordance, we carry out a detailed analysis situating each in the context of Ostrom's principles, considering both the potentials of algorithmic governance and the importance of incorporating communities' social practices into blockchain-based tools to foster forms of self-governance. The relationships found between these affordances and Ostrom's principles allow us to provide a perspective focused on blockchain-based commons governance.
\end{abstract}

\section{Keywords}

algorithmic governance, blockchain, commons governance, commons-based peer production, decentralization

\section{Introduction}

The growth of the blockchain development ecosystem has encompassed the rise of a new generation of applications and capabilities which surpass those of cryptocurrencies. We can find applications beyond Finance in multiple sectors (Hassan et al., 2020), including supply chains, energy, internet of Things or, notably, governance. In this article, we draw on the work of the Nobel laureate economist Ostrom (1990) to focus on the relationship between blockchain properties and the generation of potentialities that could facilitate governance processes. Ostrom's research showed that under certain conditions resources can be managed in a sustainable way by local communities of peers. Her work, therefore, enables us to reflect on the use of blockchain technologies to foster experimentation with new forms of blockchain-based governance in ways that go beyond markets and public administration. With this purpose, we develop from classic studies on the organizational aspects of how commons are governed and evaluate the potential use of blockchain technologies in this context. The purpose is twofold: on one hand, this work aims to throw some light on the current - and often polarized - theoretical discussions concerning both the transformative potentials of blockchain, and the consideration of emerging decentralized technologies to facilitate a new generation of commons-oriented communities. On the other hand, this analysis expects to facilitate the development of blockchain-enabled software tools which rely on commons-oriented principles, with practical examples to draw inspiration from.

In the case of blockchain-related technologies, we are still witnessing the early stages, and thus their future potential is just starting to be explored. The first cryptocurrency based purely on a peer-to-peer system, Bitcoin, was presented in

'Complutense University of Madrid, Spain

${ }^{2}$ Harvard University, Cambridge, MA, USA

\section{Corresponding Author:}

David Rozas, GRASIA Research Group, Knowledge Technology Institute, Faculty of Computer Science, Complutense University of Madrid, C/ Profesor José García Santesmases, 9, Madrid 28040, Spain. Email: drozas@ucm.es 
November 2008 in a paper published pseudonymously (Nakamoto, 2008). For the first time, no third parties were necessary to solve problems such as double-spending, while providing a novel consensus method. The solution was achieved through the introduction of a data structure known as a blockchain. In simple terms, a blockchain can be understood as a distributed and append-only ledger. Data, such as the history of transactions generated using cryptocurrencies, can be stored in a blockchain without the need to trust a third party, such as a bank server. Thus, blockchain enables the implementation of novel properties at an infrastructural level in a fully decentralized manner.

The first wave of blockchain technologies (2009-2013) starts with the advent of Bitcoin and the subsequent emergence of a broad range of cryptocurrencies ("altcoins"). The second wave (2014-today) is the extension of these blockchains with capabilities beyond currencies, that is, automatic agreements or complex tokens. These blockchains (most notably, Ethereum ${ }^{1}$ ) have introduced the ability to upload small snippets of code, so-called smart contracts (Szabo, 1997), directly onto the blockchain. Clauses are encoded in a manner by which they are automatically enforced and executed without the need for a central authority.

Thus, a complex set of smart contracts may be set up in such a way as to make it possible for multiple parties to interact with each other. This has enabled the emergence of a new kind of organization: the Decentralized Autonomous Organization (DAO). A DAO is a blockchain-based system that enables people to coordinate and self-govern themselves mediated by a set of self-executing rules deployed on a public blockchain, and whose governance is decentralized (i.e., independent from central control; Hassan \& De Filippi, 2021). This may be understood as analogous to a legal organization, with legal documents that define the rules of interaction among members. Similarly, the DAO members' interactions are mediated by the rules embedded in the DAO code. And such rules are automatically enforced by the underlying technology: the blockchain.

Commonly associated with cryptocurrencies, the untapered potential of blockchain lies in its capacity to enable the implementation of novel properties at an infrastructural level in a fully decentralized manner. The properties most cited include immutability, transparency, persistency, resilience, and openness (Underwood, 2016; Wright \& De Filippi, 2015). There have been other decentralized technical infrastructures with varying degrees of success which also reflect some of these properties, for example, the Web has been traditionally shown as an example of openness, although with varying persistence (Koehler, 1999), or BitTorrent peer-topeer sharing networks are considered open, resilient, and partially transparent (Cohen, 2003). However, none of the existing decentralized technologies have enabled the presence of all these properties at once in a robust manner, while maintaining a high degree of decentralization. It is precisely this possibility of developing technological artifacts that rely on a fully decentralized infrastructure that has been generating enthusiasm, or "hype" according to some authors (Reber $\&$ Feuerstein, 2014), with regards to the potential applications of blockchain. It is worth noting that despite the promises, "full decentralisation" is a goal that is not fully realized by actual blockchain implementations (e.g., Beikverdi \& Song, 2015).

In this article, we focus on some of these potential applications of blockchain. More precisely, we reflect on the relationship between blockchain properties and the generation of potentialities which could facilitate governance processes. Particularly, we focus on the governance of Commons-Based Peer Production (CBPP) communities. There are multiple examples of this phenomenon in a broad range of areas (Salcedo \& Fuster-Morell, 2014), including well-known projects such as Wikipedia, a project to collaboratively write a free encyclopedia; OpenStreetMap, a project to create free/ libre maps of the World collaboratively; Stack Exchange, which are Q\&A communities which aim to provide accessible documentation; Thingiverse, which provides open 3D-printable digital designs; or Free/Libre Open Source Software (FLOSS) projects such as the operating system GNU/Linux or the browser Firefox.

The term CBPP, originally coined by Benkler (2002), refers to a model of socioeconomic production in which groups of individuals cooperate with each other to produce shared resources without a traditional hierarchical organization (Benkler, 2006). The mode of production of CBPP has been characterized as decentralized (Arvidsson et al., 2017; Forte et al., 2009; Rozas \& Huckle, 2021), meaning there is a lack of a central organizing authority that coordinates the contribution of individual agents, which are instead able to collaborate without such centralized control. These two characteristics of CBPP - decentralization and the frequent use and production of shared resources - led us to explore the role of blockchain technologies in the context of supporting the coordination efforts of CBPP communities. This debate is frequently discussed from polarized approaches from both blockchain idealists and skeptics (as seen in section "Potentials of Blockchain for Commons Governance"). Meanwhile, it is emerging a growing ecosystem of blockchain projects aiming to contribute to the "social good" through peer-to-peer communities, although typically lacking awareness of how to appropriately support CBPP (Hassan et al., 2020). Thus, we aim to contribute to the ongoing debate with an analysis of which affordances blockchain technologies generate that may facilitate the governance ${ }^{2}$ of, specifically, CBPP communities. We hope that this analysis will facilitate the implementation of new blockchain projects specifically designed to support these commons-oriented communities.

This article is structured as follows: The section "Potentials of Blockchain for Commons Governance" reviews the main standpoints on blockchain-based governance ${ }^{3}$ and the section "Ostrom's Principles: Beyond Markets and Public Administration" provides an overview of Ostrom's principles 
employed to carry out our analysis. The section "Affordances Generated by Blockchain for Commons Governance" places our argument in the context of a set of identified affordances drawing on Ostrom's principles: tokenization, self-enforcement and formalization of rules, autonomous automatization, decentralization of power over the infrastructure, increasing transparency, and codification of trust. We conclude, in section "Discussion and Concluding Remarks," providing a discussion of the contribution provided by the identification of these affordances as a result of bringing together literature on CBPP and blockchain-based governance.

\section{Potentials of Blockchain for Commons Governance}

The use of blockchain technologies to facilitate governance processes is beginning to attract the attention of social scientists (Risius \& Spohrer, 2017). The emergent literature revolves around speculation on whether blockchain technologies could foster the experimentation and rise of new forms of blockchain-based governance.

Two confronting standpoints dominate the emergent debate on blockchain and governance. On one hand, there are perspectives characterized by a high degree of technodeterminism. These perspectives envisage the emergence of new forms of blockchain-based governance on the basis of the potential of these technologies for decentralization and trustlessness. These discourses inherently embed the idea of "market" and tend to ignore the complexity of social organization. For example, they commonly assume that hierarchies between the participants in decision-making processes vanish: thanks to the disintermediation enabled by blockchain technologies (e.g., Hayes, 2016; Heuermann, 2015; Swan, 2015). Overall, they tend to provide reductionist accounts with regards to the distribution of power, failing to acknowledge issues such as the generation of oligarchies (De Filippi \& Loveluck, 2016; Freeman, 1972; Shaw \& Hill, 2014). These techno-determinist perspectives are not new, nor a particular issue for blockchain technologies: they resemble, for example, the techno-determinist discourses during the popularization of access to the internet in the 1990s (Wellman et al., 2006). Still, they seem to be reinvigorated from the multiple scenarios that blockchain technology brings, as an exemplification of Hayek-like libertarian views (Bodon et al., 2019).

On the other hand, a critical stand against these technodeterminist perspectives has successfully identified and criticized the limitations of such approaches (e.g., Atzori, 2015; Atzori \& Ulieru, 2017). Nevertheless, this critique is built upon the reinforcement of the role of central authorities, resembling traditional responses against unregulated markets. In other words, these views consider traditional central authorities as inherently necessary to enable democratic governance and, as a result, ignore the potential for communities, such as the aforementioned CBPP communities, to successfully self-organize. By drawing on this assumption, the potentialities of blockchain are envisioned in nontransformative ways: to support the control required by traditional centralized forms of governance, for example, providing more transparency to their central institutions (Nguyen, 2016) or more efficient mechanisms to avoid tax fraud (Ainsworth \& Shact, 2016).

In this article, we reflect on the extent to which it would be feasible to incorporate into the development of blockchain-based tools principles from commons governance. We contribute a perspective which neither relies on the logics of private markets, as implicitly assumed by these former perspectives, nor on the coercion of traditional centralized institutions, as in the case of the latter accounts. To this end, we bring together the literature on governance of CBPP for the emerging debate on these new forms of blockchain-based governance.

Recently, a few authors have attempted to link the commons with blockchain capabilities, either at a general conceptual level (Bollier, 2015; Davidson et al., 2018; O’Dwyer, 2015) or proposing specific theoretical systems (Cila et al., 2020; Ducrée et al., 2020; Pazaitis et al., 2017). Some relevant attempts include Calcaterra (2018), who hastily mentions how Ostrom's governance principles could be applied to DAOs, and Shackelford and Myers (2017), who review the applicability of these principles focusing on governance of blockchains (instead of by blockchains). Other authors, without mentioning blockchain, consider how Ostrom's principles could be formalized and mathematized (Pitt et al., 2012, 2017), or applied to algorithmic governance (Clippinger \& Bollier, 2014).

In contrast, in this article, we will perform a detailed and systematic analysis of the affordances of blockchain for CBPP community governance which brings previous literature on organizational aspects of CBPP together with the emerging literature on blockchain-based governance. In other words, we explore functional and relational aspects that, while not determining, shape and frame the possibilities for agentic action of CBPP communities with respect to the blockchain (Hutchby, 2001). This approach is in line with previous studies of technical affordances in the study of the internet (Wellman, 2004), social media (boyd, 2010), and social movements (Juris, 2016), to name but a few examples. Thus, the aim of this article is to study which affordances blockchain technologies generate that may facilitate the governance of CBPP communities.

With this aim, we develop from classic studies on the organizational aspects of commons governance, and evaluate the potential use of blockchain technologies in this context. More specifically, we contribute to this discussion by drawing on the work of the Nobel laureate economist Ostrom (1990), whose research showed that under certain conditions commons can be managed in a sustainable way by local communities of peers. Her work, therefore, enables us to reflect on the use of blockchain technologies to foster experimentation with new forms of blockchain-based governance in ways that 
go beyond markets and public administration. As mentioned above and expanded in section "Discussion and Concluding Remarks," we aim to contribute with a fresh perspective on the often polarized debate on the transformative power of blockchain, while facilitating the building of new software tools that seek to rely on commons-oriented approaches.

\section{Ostrom's Principles: Beyond Markets and Public Administration}

Ostrom's studies focused on how communities manage to successfully govern communal resources by revisiting Hardin's (1968) influential article on "The tragedy of the commons." In this article, Hardin states how resources shared by individuals acting as homo-economicus, that is, out of self-interest to maximize their own benefit, results in the depletion of the commons. The individuals' interests enter into conflict with the group's, and because they act independently according to their short-term interests, the result of the collective action depletes the commons. As a consequence, the traditional view was that to avoid this logic, it was necessary to manage these commons through either private ownership or public administration. Parallels can be found between these standpoints and those previously summarized with regards to the emergent discussion on new forms of blockchain-based governance: they envision forms of governance which either rely on markets or on traditional forms of public administration.

Refuting Hardin's argument, Ostrom's work shows how, under certain conditions, commons can indeed be managed in a sustainable way by local communities of peers. Her approach takes into account that individual agents do not operate in isolation, nor are they driven solely by self-interest, that is, beyond homo-economicus approaches. Instead, she argues that communities communicate to build common protocols and rules that ensure their sustainability. This hypothesis was strongly supported by a meta-analysis of a wide range of case studies of communities managing as diverse resources as fisheries or irrigation infrastructure (Ostrom, 1990), and has been confirmed in later research (Cox et al., 2010; Ostrom, 2009). Furthermore, her work was subsequently employed to understand how communities develop and maintain digital commons (e.g., Fuster-Morell, 2010; Hess, 2008; Hess \& Ostrom, 2007), such as Wikipedia (Forte et al., 2009; Viégas et al., 2007) and Free/Libre Open Source Software (Rozas, 2017), and even to understand how online communities share copyrighted materials through P2P networks avoiding free-riding (Harris, 2018). As part of this work, she identified a set of principles (Ostrom, 1990) for the successful management of these commons:

1. Clearly defined community boundaries: to define who has rights and privileges within the community, for example, to use certain resources or to perform certain actions on them.
2. Congruence between rules and local conditions: the rules that govern behavior or commons use in a community should be flexible and based on local conditions that may change over time. These rules should be intimately associated with the commons, rather than relying on a "one-size-fits-all" regulation.

3. Collective choice arrangements: to best accomplish congruence (Principle number 2), people who are affected by these rules should be able to participate in their modification, and the costs of alteration should be kept low.

4. Monitoring: some individuals within the community act as monitors of behavior in accordance with the rules derived from collective choice arrangements, and they should be accountable to the rest of the community.

5. Graduated sanctions: community members actively monitor and sanction one another when behavior is found to conflict with community rules. Sanctions against members who violate the rules are aligned with the perceived severity of the infraction.

6. Conflict resolution mechanisms: members of the community should have access to low-cost spaces to resolve conflicts.

7. Local enforcement of local rules: local jurisdiction to create and enforce rules should be recognized by higher authorities.

8. Multiple layers of nested enterprises: by forming multiple nested layers of organization, communities can address issues that affect resource management differently at both broader and local levels.

Over the course of the next section, we draw on these principles to identify affordances generated by blockchain technologies which could foster, limit, or shape the governance of communities which collectively manage and produce commons.

\section{Affordances Generated by Blockchain for Commons Governance}

We incorporate the new generation of blockchain technologies and identify a set of affordances ${ }^{4}$ (Hutchby, 2001), understood as the potential uses and applications these technologies enable. Each affordance is situated in the context of commons governance drawing on the aforementioned principles of Ostrom's work. ${ }^{5}$ Table 1 provides a summary of the relationships between these affordances and Ostrom's principles.

To extract the affordances we have listed, we have aimed to cover the main properties of blockchain found in the literature, while focusing on those relevant for governance by blockchains (Ølnes et al., 2017), that is, the organization processes of communities which rely at least partially on blockchain infrastructure (e.g., an organization using a blockchain voting application to approve and fund a project), as opposed to governance of blockchains, that is, the organization processes of developers to 
Table I. Summary of the Relationships Between the Identified Affordances of Blockchain Technologies for Governance and Ostrom's (1990) Principles.

\begin{tabular}{|c|c|c|c|c|c|c|}
\hline Affordancelprinciple & Tokenization & $\begin{array}{l}\text { Self-enforcement } \\
\text { and formalization }\end{array}$ & $\begin{array}{l}\text { Autonomous } \\
\text { automatization }\end{array}$ & $\begin{array}{l}\text { Decentralization } \\
\text { of power over } \\
\text { infrastructure }\end{array}$ & $\begin{array}{l}\text { Increasing } \\
\text { transparency }\end{array}$ & $\begin{array}{l}\text { Codification } \\
\text { of trust }\end{array}$ \\
\hline $\begin{array}{l}\text { I. Clearly defined community } \\
\text { boundaries }\end{array}$ & $\checkmark$ & & & & & \\
\hline $\begin{array}{l}\text { 2. Congruence between rules and } \\
\text { local conditions }\end{array}$ & $\checkmark$ & $\checkmark$ & & $\checkmark$ & & \\
\hline 3. Collective choice arrangements & $\checkmark$ & & & $\checkmark$ & & \\
\hline 4. Monitoring & & $\checkmark$ & $\sqrt{ }$ & $\sqrt{ }$ & $\checkmark$ & \\
\hline 5. Graduated sanctions & & $\checkmark$ & $\checkmark$ & & & \\
\hline 6. Conflict resolution mechanisms & & & $\checkmark$ & & $\checkmark$ & \\
\hline 7. Local enforcement of local rules & & $\checkmark$ & & $\sqrt{ }$ & & $\checkmark$ \\
\hline 8. Multiple layers of nested enterprises & & & $\sqrt{ }$ & & & $\checkmark$ \\
\hline
\end{tabular}

build and evolve blockchains and their rules (e.g., the rules that check if that a transaction is valid in a cryptocurrency such as Bitcoin). The blockchain properties most cited include immutability, transparency, persistency, resilience, and openness (Underwood, 2016; Wright \& De Filippi, 2015). However, properties concerning DAOs are also relevant to blockchain governance, even if not a property of blockchain itself. Taking these issues into account, we have decided to group the blockchain properties relevant to governance by blockchains into six affordances: (a) tokenization, or how blockchain facilitates the creation and management of tokens (Cong et al., 2020; Lo \& Medda, 2020); (b) self-enforcement and formalization, referring to the self-enforcing capabilities of smart contracts, which facilitate formalizing rules as code (De Filippi \& Hassan, 2016); (c) autonomous automatization, or how DAOs present new capabilities and challenges (DuPont, 2017); (d) decentralization of power over infrastructure, or how decentralized technologies enable new power dynamics between social and technical power (Forte et al., 2009); (e) increasing transparency, relying on the persistency and immutability properties which enable all users to access the blockchain data (De Filippi, 2018); (f) codification of trust, one of the most cited properties of blockchain, which supposedly enables "trustless" systems (Werbach, 2018).

To illustrate the identified affordances, we use a recurring example. We select a specific type of CBPP: a community network. In these communities, participants provide and manage technical infrastructure as a common resource to provide internet access. Examples of these communities include Guifi.net, ${ }^{6}$ Ninux, ${ }^{7}$ or Sarantoporo. ${ }^{8}$ Usually, these communities involve complex governance including online and offline interactions at several levels of organization, from local nodes to umbrella communities.

\section{Tokenization}

An essential feature of blockchain technologies is their capacity for tokenization. Tokenization refers to the process of transforming the rights to perform an action on an asset into a transferable data element (named token) on the blockchain. For example, in the medical field, tokenization has been employed to provide authorization regarding access to reports (Azaria et al., 2016; Liu, 2016).

In the Bitcoin blockchain, the term token is used as an abstraction of the actual "coin," that is, the cryptocurrency being transferred among users. The rise of blockchain-based cryptocurrencies is a product of such a feature because blockchain's facility for the creation, transfer, and management of tokens in a distributed manner is unparalleled. This process of tokenization facilitates the distribution of value and incentives. Third parties, such as banks or gateways, are not necessary to transfer value between individuals or across networks. Furthermore, such tokens may be used as more than holders of monetary value: they may represent equity, decision-making power, property ownership, or labor certificates $^{9}$ (Huckle \& White, 2016). This capacity for tokenization of blockchain technologies provides a series of affordances for technological artifacts constructed to facilitate governance. In the context of CBPP communities, tokenization relates to several of Ostrom's principles.

Ostrom's first principle states the importance of the definition of community boundaries for governance. These boundaries are reflected in the rules embedded in the software employed to coordinate communal activity in CBPP. This software typically defines permissions or rights to access or modify resources or community rules. In such a context, we can envision the use of tokens to construct tools, in which participation rights can be more easily and granularly defined, propagated, and/or revoked. For example, in the case of a community network, access to the infrastructure could be granted with tokens, for example, those people who have contributed enough infrastructure, or paid the agreed price, could access the internet through the community network. This specific use of blockchain has been proposed by Guifi.net, one of the largest, most prominent community networks (Kabbinale et al., 2019). 
Negotiations regarding the definition of boundaries and their reflection in the technical artifacts connect additionally to the second and third principles of Ostrom. CBPP communities require constant processes of development of collective choice arrangements regarding their governance (e.g., Forte et al., 2009; Rozas, 2017; Schweik \& English, 2013). They define rules based on local conditions, and seek to find ways in which those affected by these rules can participate in their modification, as understood in the second and third of the principles. For instance, to compensate contributions, Guifi.net differentiates between volunteers and professional actors, and further categorized professional actors depending on their level of commitment (from full to opportunistic).

Overall, the capacity for tokenization of blockchain technologies could be employed to readdress latent power relations in these communities. Negotiations in these communities, while maintaining a social character, would be mediated by blockchain-based artifacts which in turn would be communally constructed. This implies an exercise by the community to specify the tasks to be carried out providing an opportunity for certain often-forgotten tasks - such as care labor (PérezOrozco, 2014) - to be made visible. That is, care tasks, such as emotional labor, conflict management, maintenance, or events organization, may be made visible and acknowledged by the community - along with those undertaking such tasks. Tokenization, therefore, provides an opportunity to rethink existing power dynamics within CBPP communities.

In this respect, some concepts from feminist economic theory-such as that of invisible labor (Pérez-Orozco, 2014) — can shed light on the usefulness of blockchain-based tools for governance. Instead of narrowing the use of tokens to grant rights to access, we consider their potential to address the imbalance of invisible labor, such as making certain forms of power more visible, an issue which tends to become more critical when CBPP communities need to scale up their self-organizational processes.

While techno-determinist discourses assume that "anything that can be decentralized will be" (Johnston, 2014), and at least partially tokenized as a result, this is a controversial view, as tokenization also presents risks. An example of these risks includes extreme quantification and data fetishism (Sharon \& Zandbergen, 2017). Thus, we must seek a balance in the limits regarding what kind of actions should or should not be tokenized, what kind of mechanisms are established to change the status quo, and how communities assess the desirable degree of tokenization in their governance. In other words, there is a need to further understand the affordance of tokenization and explore how self-organized communities may or may not incorporate it into the technological artifacts employed for collaboration and to what extent.

\section{Self-Enforcement and Formalization of Rules}

Blockchain entails an affordance for self-enforcement and formalization of rules which are intertwined with Ostrom's principles. Examples of these rules are those which regulate monitoring and graduated sanctions, as reflected in Ostrom's fourth and fifth principles. Blockchain technologies could partially embed some of these governance rules in technological artifacts. Scenarios in which communities define certain rules regarding the allocation of common resources - through actions such as pooling, capping, or mutualizing - and in which these rules are automatically enforced can be envisioned. Following previous examples, one can imagine a capping rule agreed by a community network which automatically enforces a previously negotiated internet bandwidth limit, or which automatically penalizes a misuse of the common network. Another example could consist of a set of self-enforced rules for a redistribution mechanism that grants internet access to those in the communities with fewer resources. It can be envisioned how at least a significant part of the monitoring could be embedded into the code, instead of requiring participants to manually perform some of these monitoring operations.

In addition, blockchain technologies require the rules to be unambiguously understood by machines. This implies a need to formalize the governance rules which are usually expressed in the inherently ambiguous natural language. Thus, this explicitation could lead to the need to discuss these rule changes to formalize and encode them. It therefore provides an affordance for formalizing rules which presents several limitations, which will be subsequently discussed, as well as a set of potentialities.

Research on how self-organization occurs in CBPP communities has shown that - counterintuitively to the initial accounts criticized by authors such as Viégas et al. (2007) or Mateos-García and Steinmueller (2008) - the changes experienced in the self-organizational processes of CBPP communities tend to show an increase in the degree of formalization around decision-making over time when they grow, which is explained as a means to achieve decentralization and to scale up communities (e.g., Forte et al., 2009; Rozas \& Huckle, 2021; Schweik \& English, 2013). This has been identified even in cases with a generally antibureaucratic attitude, such as communities with a strong hacker culture which aim to avoid formal and bureaucratized systems (Rozas, 2017). Thus, the process of explicitation of rules which is encompassed in the development of smart contracts related to the use of distributed technologies also provides opportunities to make these rules more available and visible for discussion, as noted in the second principle of Ostrom. Furthermore, formalization in combination with self-enforcement relates to the seventh principle of Ostrom: local nodes of CBPP communities could more easily ensure that the local jurisdiction ${ }^{10}$ and enforcement of local rules is acknowledged by higher authorities or by other nodes.

For example, an organizational structure of a large community network in which a set of local nodes are federated, and each node possesses local autonomy to develop its own rules regarding the management of the local infrastructure. A node 
might be based in Madrid and another in Berlin. Rules can be established in which the autonomy to take decisions regarding the node in Madrid belongs, by code, to the participants of that node, and vice-versa. Furthermore, if higher authorities exist in this context, such as a European federation of nodes, to continue with our example, we can imagine rules which are selfenforced by code to ensure that the local aspects are only decided by participants of the local nodes. Overall, blockchain technologies provide affordances to foster the formalization and enforcement of this type of agreement.

Several issues, however, require further exploration with regards to the affordances of self-enforcement and formalization in the context of governance of CBPP communities. First, rules embedded in smart contracts rely on an ex-ante nature, rather than ex-post (De Filippi \& Hassan, 2016). Instead of third parties or community members monitoring and enforcing them, the rules would be automatically enforced according to agreements previously negotiated by the community. While this theoretically increases the difficulty to breach them, it also presents problems with regards to the difficulty to define exceptions (De Filippi \& Hassan, 2016). Ongoing recent blockchain projects, such as DAOStack ${ }^{11}$ or Aragon, ${ }^{12}$ provide the potential to more easily upgrade the rules embedded in smart contracts over time, in congruence with the second principle of Ostrom (congruence between rules and local conditions). Thus, this increasing capacity for upgradability which is being developed in the new generation of blockchain technologies could help incorporate these exceptions over time. However, even if a rule is updated after reaching an agreement in the community, the original code will have been applied and the new rules will only be applicable the next time. For instance, continuing with the example of community networks, a person could lose internet access due to a strict community rule that is later relaxed. From these limitations, we foresee at least two questions which require further empirical research: What are the consequences for CBPP communities of moving from ex-post forms of regulation toward ex-ante? Which aspects should remain in/off the blockchain, or further completely in/out of code?

Second, the process of formalization of these rules requires, at least with the most current technology, a high degree of technical knowledge in the translation of these rules into source code. Thus, while formalization might help make these rules more visible and available for discussion in the community, the power to specify these rules may now be shifted to those coding them. In this context, it is necessary to consider the biases - such as gender, race, and class (Platero, 2014) - of those possessing this technical knowledge. Another issue to be considered is the tendency toward accommodation or less reflexivity over time as a consequence of automation (De Filippi \& Hassan, 2016).

Third, in a similar way as with the risk of extreme tokenization presented in the previous section, there is a risk of extreme formalization in the rules that regulate the behavior of participants in these communities. The effects are unknown. Ostrom's work highlighted, for example, the relevance of informal social norms (Ostrom, 2000) for the successful selfmanagement of resources. The effects of an excessive formalization of norms into explicit rules self-enforced "by code" might become a source of distortions within the dynamics of the communities.

\section{Autonomous Automatization}

DAOs present multiple, unparalleled characteristics. The level of autonomy of these pieces of code surpasses all forms of autonomous software agents (Franklin \& Graesser, 1997). Because DAOs do not rely on central servers, DAOs cannot be shut down, unless explicitly programmed in their code. Thus, they are fully autonomous including with respect to their creator, and they function as long as a user (human or software) continues to interact with them. This may prevent censorship and the halt of malicious code, for example, a virus. In addition, DAOs may interact as autonomous users in the network, holding tokens and assets, or purchasing services from other DAOs. In fact, they can even hire users to perform tasks for them, and sell their own services or resources to third parties. Hence, individuals can transact with a DAO to benefit from the service it provides, or to be paid for a contribution. Thus, DAOs may be self-sufficient, to the extent that they can charge users for their own services (or assets) to pay for the services they need (De Filippi \& Hassan, 2016).

There is already an emerging ecosystem of DAO examples (El Faqir et al., 2020), of which may mention a few examples: the venture capital fund with the (confusing) name, "TheDAO," 13 which was one of the earliest examples; the prediction market, Augur; $;{ }^{14}$ the digital assets platform focused on gold assets, Digix,${ }^{15}$ or the decentralized exchange with a stable coin, MakerDAO. ${ }^{16}$ As it is true for the vast majority of projects in the blockchain field, they are directly related to finance, although there are already some nonfinancial examples such as the virtual world Decentraland, ${ }^{17}$ or the job market Ethlance. ${ }^{18}$ These DAOs are designed to work in a decentralized manner without central intermediaries, yet their governance model is strictly market-driven. For instance, in TheDAO, voting power was correlated to the number of tokens possessed, that is, it works as a plutocracy, controlled by the wealthy minority (as opposed to a democracy).

However, DAOs provide new possibilities with regards to CBPP. In fact, scenarios in which DAOs aid several of Ostrom's principles can be conceived. As mentioned in the previous section, smart contracts may help in the monitoring and application of sanctions for those violating the community rules (fourth and fifth principles). When DAOs are considered, this feature is strengthened because communities may rely on an automated entity for such monitoring and sanctioning. The agency of this entity, which may take the initiative and react upon circumstances, may have multiple 
implications. On one hand, its impersonalization may be positive to see that sanctions come from a community decision, preventing the common effect of reacting against the enforcer ("killing the messenger"). On the other hand, the same impersonalization may trigger frustrations and impotence (Frost \& Postman, 1993) similar to the reactions against machines.

DAOs may also contribute to higher degrees of automatization of the processes in communities, facilitating scaling up and thus the creation of layers of nested entities, as the eighth principle states. Because we are aware that scaling up communities involves an increase of formalization and bureaucratization (e.g., Forte et al., 2009; Rozas, 2017; Schweik \& English, 2013), a higher degree of automatization of processes could reduce the burden of bureaucracy, accelerate processes, and facilitate scaling up. For instance, in a community network with multiple nodes, it is common to have multiple spaces for coordination, monitoring, verification, or transfers of value and resources.

Despite clear rules, the need remains for humans to carry out multiple actions. Many communities rely on software to automate parts of this process, although this implies either governance of such software/infrastructure, or dependence on third parties and their rules for their inner processes. In such a context, a DAO can be set up to facilitate interaction and coordination across nodes. Once the rules are agreed and clear, they can be embedded in the DAO code, which can automate a large proportion of the processes, monitoring the nodes' actions, facilitating coordination, even transferring value and resources in relation to the nodes' contributions. In fact, this may be scaled up easily, with DAOs coordinating other "smaller" DAOs. Also, if other communities have their own DAOs, it may be easier to establish cooperation across communities.

To continue with previous examples, we could expect collaboration among different community networks, granting internet access to all members of any other community. These communities could share information about unconforming users to prevent network abuses and could even negotiate exchanges to account for the differences in use of the networks, scaling the compensation mechanisms that already exist within these communities.

Finally, DAOs provide a space in which governance is digitalized and formalized, and where most organizational processes should be tackled in some way, including conflicts. That is, governance formalization demands an exploration of the potential conflicts which may occur, and their possible resolution. This is directly related to Ostrom's sixth principle. Combined with the aforementioned automatization and scaling up, we may observe a space in which conflicts are made explicit, between members of a DAO, across DAOs, and between DAOs and humans. This encourages communities to establish clear mechanisms for conflict resolution, which may be at least partially tackled by automated processes. In fact, projects such as Aragon ${ }^{19}$ are already working on creating digital jurisdictions for conflict resolution within, and across, DAOs. Moreover, community networks such as Guifi.net already use conflict resolution systems similar to these proposals, standardizing how conflicts should be resolved aiming to reduce the time and increase the scalability of conflict resolutions (Baig et al., 2015).

There are, however, some shortcomings of this affordance. Indeed, such a "DAO world" has multiple potentials, and yet, it is worth remembering that DAOs are constrained to the digital world. That is, digitalization is expanding quickly and affecting the physical world in multiple ways, and yet the physical world continues to operate with its own rules. Although techno-determinist views often disregard this fact, humans have bodies, which are constrained by their physical reality, and cannot be ignored or "disappear" in cyberspace (Le Breton, 2015). Thus, DAOs may allow digital voting, but a DAO cannot know if a person is being coerced to vote in a certain way. DAOs may allow the transfer of digital assets, and yet laptops can be stolen.

In the same vein, DAOs may hire services or resolve conflicts, and yet there is a legal framework that humans are subject to that may contradict the DAOs' decisions. In fact, DAOs open up multiple unresolved challenges with respect to law (De Filippi \& Wright, 2018). For instance, on liability, they are as follows: Who is liable for a DAO misaction, such as the loss of money? The creator of the DAO, who may not control it? The members of the DAO, who could influence its evolution? The project managing the blockchain where the DAO operates? Or is it worth considering the DAO itself as a subject of liability?

Summing up, the use of DAOs for commons governance remains speculative, and it may imply challenges and risks. However, multiple opportunities may arise from using these new "agents" as automatic helpers for communities, which would enable the automatization of bureaucratic processes, facilitate scaling up, and making conflict resolution mechanisms more explicit.

\section{Decentralization of Power Over Infrastructure}

This affordance refers to the process of communalizing the ownership and control ${ }^{20}$ of the technological artifacts employed by the community through the decentralization of the infrastructure they rely on.

This affordance can be illustrated when exploring the relationships between technical and social power (Forte et al., 2009) which occur in CBPP communities together with the forms of pressure which surround them. The control over the infrastructure that sustains, for example, the main platforms of collaboration, commonly emerges as a point of tension and conflict. When CBPP communities start to grow substantially, they normally try to decentralize control over this infrastructure, which is commonly achieved by incrementing the degree of formalization, for example, defining more explicit and rigid organizational processes, roles and 
even formal institutions, such as identified for Wikipedia (Forte et al., 2009) or FLOSS communities (Rozas, 2017). These organizational changes entail constant negotiation which, when framed through Ostrom's principles, can be understood as part of the generation of collective choice arrangements (third principle) and do not commonly occur in a scenario of equality in terms of power.

The use of decentralized technologies offers, in this respect, a promising field of experimentation and exploration of potential changes in the relationships between technical and social power. An illustration can be found in the "right to fork" which, while it may be perceived as an aspect unique to FLOSS communities, has indeed been identified in other CBPP communities (Jemielniak, 2016; Tkacz, 2014). The inherent properties of decentralized technologies facilitate the forking of the whole infrastructure and, as seen, even the communitarian rules encoded in smart contracts. In other words, those in control of the infrastructure might not only fear the forking of the contents (e.g., source code or wiki pages), but of the whole infrastructure and a large set of the codified community rules. These examples allow us to imagine scenarios of the possible opportunities gained by decentralizing power over infrastructure in CBPP. Decentralized technologies may shape these dynamics by offering a higher degree of pressure for negotiation on those holding more power in the community and fostering permissionless innovation (Thierer, 2016).

Continuing with our community network example, part of the centralized infrastructure - such as that related to monitoring and compensating imbalances in the uses of the shared infrastructure-could be decentralized (Rozas, 2020). Community networks, such as Guifi.net, have developed compensation systems as part of their governance which relate to several of Ostrom's principles (Baig et al., 2015). Decentralization of the infrastructure reduces the technical cost to fork the infrastructure, reducing the power within the community of those previously in control of it.

When we analyze this affordance through Ostrom's principles, we identify a set of aspects which relate to them. First, those holding more power within the community may experience higher pressure with regards to the constant processes of negotiation of collective choice arrangements - the third principle. Second, in connection with the fourth principle of Ostrom, those monitoring the commons could also experience new forms of pressure regarding their expected accountability in the eyes of the community. Third, within this scenario, the decentralization of power over infrastructure could facilitate permissionless innovation and thus a higher degree of autonomy ${ }^{21}$ to the local spaces which emerge over time. Thus, the differences in the forms of pressure may provide new conditions for the negotiations that relate to having their local contexts and jurisdictions acknowledged by higher authorities - in congruence with the second and seventh principle of Ostrom, respectively.

Nevertheless, the affordance for decentralization of power over infrastructure is not free of risks. A risk that can be expected is a shift of power to those coding the rules, as previously discussed for the cases of tokenization, self-enforcement, and formalization of rules. In addition, the aforementioned higher degree of pressure for negotiation or permissionless innovation could result in increasing risks of the constant fragmentation of the community. The issue is not new. Large CBPP communities, for example, constantly aim to navigate these tensions to "loosen control without losing control" while trying to scale up (Rozas \& Huckle, 2021). The key resides in furthering our understanding on how to integrate this affordance for decentralization of power over the infrastructure into the dayto-day practices of these communities.

\section{Increasing Transparency}

Increasing transparency refers to the process of opening the organizational processes and the associated data by relying on the persistency and immutability properties of blockchain technologies. Blockchain enthusiasts envision a blockchain governance as one that "takes advantage of the public recordkeeping features of blockchain technology: the blockchain as a universal, permanent, continuous, consensus-driven, publicly auditable, redundant, record-keeping repository" (Swan, 2015, p. 44).

Blockchain technologies provide a potential for CBPP communities to socially construct software in which certain actions and operations are more easily trackable, auditable, and communally fiscalized by their participants. CBPP communities have, indeed, a long tradition of aiming to make their processes as open and participative as possible. Examples of these data are the materials generated as a result of encounters when decisions are made, or the indicators of the degree of participation in the community. This strong culture of openness and participation in CBPP communities connects with the fourth and sixth principles of Ostrom (monitoring and conflict resolution). The opening of the data generated in the collaboration processes in the communities is a useful means by which CBPP communities successfully carry out and scale up their processes of monitoring. They increase the legitimacy of these processes and provide means of accountability for those who participate in them in the eyes of the community. These data are also commonly employed as part of conflict resolution mechanisms as well as in the constant processes of negotiation. One can think, for example, of the enormous amount of contents which can be found in the discussion pages of Wikipedia or in the issues lists of FLOSS communities. These large amounts of data are not solely related to the contents but also to the organizational processes themselves.

The experimentation with software drawing on blockchain technologies provides new possibilities for CBPP communities to track and communally fiscalize new aspects of their processes. Continuing with the example of community networks, this transparency can help identify who uses more resources, the community can then either try to grant these resources or to penalize excessive usage; those who 
contributed more can also be rewarded or recognized accordingly. For instance, in the case of the aforementioned compensation system of Guifi.net (Baig et al., 2015), it would facilitate the monitoring beyond central points of control (Rozas, 2020).

As with the previously discussed affordances, however, commons-based approaches toward the use of blockchainbased tools for governance should be aware of the limitations. Khan (2017), for instance, places this into the more general discussion of privacy and the right to be forgotten in the digital age (Mayer-Schonberger, 2009). The permanent nature of blockchain opens up scenarios in which "everything is recorded" and "will forever tether us to all our past actions, making it impossible, in practice, to escape them" (Rosen, 2010). Extreme transparency in the context of self-governance of CBPP communities raises similar questions: What kind of participation information should be permanently stored? Or, how might a scenario with a higher degree of transparency shape the development of participants' identities in the communities?

\section{Codification of Trust}

Trustlessness is one of the most cited characteristics by blockchain enthusiasts to argue for the disruptive potential of this technology. When framed in terms of processes, it can be understood as that of codifying trust into "trustless systems" developed under a blockchain. In simple terms, trustless systems are those which enable participants to enter into an agreement, without requiring a third party to provide a certain degree of trust between them.

Commons-based approaches require a re-interpretation of "trustlessness" as a partial property, however, which may act as a potential source of affordances in the context of commons governance. An example of these limitations relates to the transfer of trust encompassed in the design and development of these trustless systems. For example, when considering the use of smart contracts to facilitate governance, trust is transferred to the code that defines them, and subsequently to those who write the code. In fact, some have characterized blockchains as a new architecture of trust (Werbach, 2018).

The codification of trust can bring interoperability into CBPP communities. In technical terms, interoperability refers to the property of a system to operate with other systems through a series of software interfaces. Blockchain provides affordances to increase the degree of collaboration through the generation of interoperable interfaces and, furthermore, providing a full communal infrastructure. In practice, blockchain has been cited as enabler of interoperable ecosystems, for instance, in Internet of Things (Reyna et al., 2018), although global standards are still rare beyond templates (such as those from Open Zeppelin), Ethereum-like Request for Comments (RFCs, that is, ERCs), and some fractioned attempts at inter-blockchain interoperability (e.g., Interledger, Polkadot).

This affordance for the codification of trust relying on a communal infrastructure allows us to imagine potentialities at several levels: first - and in connection with the seventh and eighth principles of Ostrom - to facilitate internal interoperability among the different groups or nodes that form part of CBPP communities, or the multiple layers of nested enterprises in Ostrom's terms.

Returning again to our previous example of a community network - with local nodes in Berlin and Madrid - one can envision artifacts designed to facilitate the governance of CBPP communities in the form of different platforms which are customized according to local conditions. These platforms could be autonomously governed by the participants who belong to each of the nodes, but interoperate between them and/or with a federal platform at a broader level. The process of codification of trust would not simply refer to the individuals and their interactions. Instead, it could include the agreements arranged between the nodes that form part of the community, fostering the capacity of these communities to scale up some of their self-organizational processes.

Second, a blockchain as a common database infrastructure generates affordances for interoperability beyond the boundaries of a particular CBPP community. For example, a set of smart contracts which encode agreements between community networks, or by reflecting the decisions made by different community networks with regards to their different notions of value (Rozas et al., 2021) and ways to make them interoperable (De Filippi \& Hassan, 2015). Nevertheless, as with the previously discussed affordances, the processes related to the codification of trust in ways that facilitate interoperability between and within CBPP communities will remain as social processes of negotiation. As such, they are not exempt from similar risks as those discussed for the previous affordances.

\section{Discussion and Concluding Remarks}

We explored blockchain as an artifact to facilitate the governance of communities, with an emphasis on CBPP communities. CBPP communities are characterized by Ostrom's (1990) principles for commons governance, and our analysis showed the different affordances that blockchain technology provides to those communities in view of these. We contribute a systematic identification of such affordances and discuss their implications.

Our study does not only highlight blockchain affordances with respect to CBPP communities, but analyzes how each individual affordance may support Ostrom's principles and discusses how community governance may be affected. Moreover, we contribute to the emergent literature on blockchain-based governance by providing a novel perspective which does not rely on techno-determinist views and logics of private markets (e.g., Hayes, 2016; Heuermann, 2015; Swan, 2015), nor on the assumption of the need for coercion by traditional centralized institutions (Atzori, 2015). Our perspective allows us to open up new questions for exploration. Examples of these research questions are those discussed with regards to the limits of the processes of the 
tokenization or formalization of rules: Which aspects should remain in/off the blockchain, or further completely in/out of code? Thus, by providing a perspective focused on commons governance, we do not simply identify the potentialities, but also possible drawbacks and limitations which are not addressed by techno-determinist views, nor identified and properly analyzed by approaches that disregard the power of the self-organization of communities.

Furthermore, the identified affordances, which emerged by bringing together literature on the governance of commons within that of blockchain-based governance, can be employed as analytical categories (e.g., Rozas, 2020; Rozas, Díaz-Molina, 2019) to co-design blockchain-based tools to facilitate cooperation and foster CBPP practices. Table 1 provided a summary of these relationships between them and Ostrom's principles.

As discussed, CBPP communities face challenges trying to decentralize their organizational processes to scale-up. The relationships that we identify informed our analysis on the impact that decentralized blockchain-based technologies can have on governance processes in these communities. Examples of issues previously identified in the literature (e.g., Forte et al., 2009; Rozas \& Huckle, 2021; Schweik \& English, 2013) that relate to the identified affordances are the need to increase the degree of formalization of their processes to provide higher degrees of legitimacy, transparency, and trust. More generally, we find that blockchain-based technologies offer potentialities to facilitate coordination, help scale up commons governance and can even be useful to share agreements and different forms of value among various communities in interoperable ways.

Moreover, this study aims to contribute not only to the theoretical debate but also to provide ground for new blockchain projects to rely on, to build appropriate tools for CBPP communities. As mentioned above, there is already an emerging ecosystem of blockchain projects aiming to contribute to the "social good" through peer-to-peer communities, although typically lacking awareness of how to appropriately support CBPP (Hassan et al., 2020). There are though a few projects which do claim to rely on commonsoriented perspectives in different degrees, and thus we believe there is potential for our contribution to be of use. Relevant examples are the Commons Stack project, ${ }^{22}$ the Backfeed project, ${ }^{23}$ or the Aragon DAO platform which claims to rely on Benkler's work. ${ }^{24}$

In fact, there are already applications of this study which go beyond its theoretical contributions. The project P2PModel ${ }^{25}$ has used this theoretical framework to inform the design and development of blockchain-based applications for established commons-oriented communities (e.g., Rozas, 2020; Rozas \& Díaz-Molina, 2019). Besides, another paper provides design guidelines for tools relying on this work (Cila et al., 2020).

In sum, this article identifies blockchain affordances and connects them to Ostrom's principles for commons governance. We contribute to the emergent debate on blockchainbased governance through a commons-based approach while also providing a basis for the essential empirical research needed to improve our understanding of the role of blockchain technologies for the governance of communities.

\section{Acknowledgments}

The authors thank Mayo Fuster, David Bollier, Maria T. Vidal, Sara Gil, Jordi Burguet, and Juan Pavón for their helpful comments and suggestions. The authors are also grateful to all the participants at the congress "Science, politics, activism and citizenship" organized by Redes CTS \& the Catalan Society for the History of Science and Technology at the University of Valencia (Spain) in May 2018. Finally, the authors thank Tabitha Whittall for her help reviewing, proofreading, and improving the structure of this article.

\section{Declaration of Conflicting Interests}

The author(s) declared no potential conflicts of interest with respect to the research, authorship, and/or publication of this article.

\section{Funding}

The author(s) disclosed receipt of the following financial support for the research, authorship, and/or publication of this article: This work was partially supported by the project P2P Models (https:// p2pmodels.eu) funded by the European Research Council ERC2017-STG (grant number 759207) and by the project Chain Community funded by the Spanish Ministry of Science, Innovation and Universities (grant number RTI2018-096820-A-100).

\section{ORCID iDs}

David Rozas (iD https://orcid.org/0000-0001-6371-0964

Antonio Tenorio-Fornés (iD https://orcid.org/0000-0001-7526-4340

\section{Notes}

1. See https://www.ethereum.org (accessed September 27, 2019).

2. By "governance" we draw on Fuster-Morell's (2014) analysis and characterization of commons governance as a complex system, which incorporates social norms and the role played by the infrastructure.

3. This article focuses on governance through or with blockchains, rather than on governance of the communities which develop and maintain blockchain projects.

4. Concretely, we consider the affordances of public permissionless blockchains, that is, blockchains which new participants can freely join and use.

5. Table 1 summarizes the relation among the affordances and Ostrom's principles.

6. See http://guifi.net (accessed September 27, 2019).

7. See http://ninux.org (accessed September 27, 2019).

8. See http://www.sarantaporo.gr (accessed September 27, 2019).

9. Similar to those described by Marx (1875).

10. In this context, we refer to jurisdiction as the area over which the members of a communitarian node have control (Sullivan, 2009).

11. See https://daostack.io (accessed September 27, 2019).

12. See https://aragon.one (accessed September 27, 2019).

13. See https://medium.com/swlh/the-story-of-the-dao-its-historyand-consequences-71e6a8a551ee for the history of TheDAO that suffered a large "hack," which influenced the blockchain ecosystem and its evolution (accessed September 27, 2019). 
14. See https://augur.net (accessed January 10, 2019).

15. See https://digix.io (accessed September 27, 2019).

16. See https://makerdao.com (accessed January 10, 2019).

17. See https://decentraland.org (accessed January 7, 2020).

18. See https://ethlance.com (accessed January 7, 2020).

19. See https://github.com/aragon/whitepaper (accessed October 3, 2019).

20. As discussed in Footnote 3, in this article, we focus our analysis on governance through blockchains. For this reason, we will not tackle the relationships between technical and social power in the underlying protocols (Atzori, 2015). Examples of these are the identification of strategies regarding the mining protocol to control the system by Eyal and Sirer (2014), and the inequalities generated by the accumulation of hashing-power.

21. The coordination of different local groups would tentatively require a higher degree of interoperability. Interoperability will be discussed in further detail as part of the affordance of codification of trust.

22. Commons Stack aims to build "commons-based microeconomies to sustain public goods through incentive alignment, continuous funding and community governance" with "web3 components" (aka blockchain components; https://commonsstack.org).

23. Backfeed is a novel system of value which relies on the blockchain and aims to aid the creation of commons-oriented ecosystems (Pazaitis et al., 2017).

24. Aragon is a platform to facilitate the creation of new DAO communities. See https://wiki.aragon.org/about/history/ for some background on their inspiration from Benkler's theories.

25. See https://www.p2pmodels.eu (accessed February 23, 2021).

\section{References}

Ainsworth, R. T., \& Shact, A. (2016, October). Blockchain (distributed ledger technology) solves VAT fraud. https://doi. org/10.2139/ssrn.2853428

Arvidsson, A., Caliandro, A., Cossu, A., Deka, M., Gandini, A., Luise, V., \& Anselmi, G. (2017). Commons based peer production in the information economy. https://www.academia. edu/29210209/Commons_Based_Peer_Production_in_the Information_Economy

Atzori, M. (2015). Blockchain technology and decentralized governance: Is the state still necessary? SSRN Electronic Journal. https://doi.org/10.2139/ssrn.2709713

Atzori, M., \& Ulieru, M. (2017). Architecting the eSociety on blockchain: A provocation to human nature. Social Science Research Network. https://papers.ssrn.com/abstract=2999715

Azaria, A., Ekblaw, A., Vieira, T., \& Lippman, A. (2016). MedRec: Using blockchain for medical data access and permission management [Conference session]. 2016 2nd International Conference on Open and Big Data (OBD). https://doi. org/10.1109/obd.2016.11

Baig, R., Roca, R., Freitag, F., \& Navarro, L. (2015). Guifi.net, a crowdsourced network infrastructure held in common. Computer Networks, 90, 150-165.

Beikverdi, A., \& Song, J. (2015, June 1-3). Trend of centralization in Bitcoin's distributed network. In proceedings of the 2015 IEEE/ACIS 16th international conference on software engineering, artificial intelligence, networking and parallel/ distributed computing (SNPD) (pp. 1-6), Takamatsu, Japan. IEEE. https://doi.org/10.1109/SNPD.2015.7176229
Benkler, Y. (2002). Coase's penguin, or, linux and "the nature of the firm." The Yale Law Journal, 112(3), 369-446.

Benkler, Y. (2006). The wealth of networks: How social production transforms markets and freedom. Yale University Press.

Bodon, H., Bustamante, P., Gomez, M., Krishnamurthy, P., Madison, M. J., Murtazashvili, I., Murtazashvili, J. B., Mylovanov, T., \& Weiss, M. B. H. (2019). Ostrom amongst the machines: Blockchain as a knowledge commons. https:// doi.org/10.2139/ssrn.3462648

Bollier, D. (2015, May 3). The blockchain: A promising new infrastructure for online commons. News and Perspectives on the Commons. http://www.bollier.org/blog/blockchain-promisingnew-infrastructure-online-commons

boyd, D. (2010). Social network sites as networked publics: Affordances, dynamics, and implications. In Z. Papacharissi (Ed.), A networked self (pp. 47-66). Routledge.

Calcaterra, C. (2018). On-chain governance of decentralized autonomous organizations: Blockchain organization using Semada. Social Science Research Network. https://papers.ssrn.com/ abstract $=3188374$

Cila, N., Ferri, G., de Waal, M., Gloerich, I., \& Karpinski, T. (2020). The blockchain and the commons: Dilemmas in the design of local platforms. In CHI '20: Proceedings of the $2020 \mathrm{CHI}$ conference on human factors in computing systems (pp. 1-14). Association for Computing Machinery.

Clippinger, J., \& Bollier, D. (2014). From Bitcoin to burning man and beyond: The quest for identity and autonomy in a digital society ID3 and Off The Common Books.

Cohen, B. (2003, June 5-6). Incentives build robustness in bittorrent [Conference session]. $1^{\text {st }}$ Workshop on Economics of Peer-to-Peer Systems (Vol. 6). Berkeley, CA, USA.

Cong, L. W., Li, Y., \& Wang, N. (2020). Tokenomics: Dynamic adoption and valuation (w27222). National Bureau of Economic Research. https://doi.org/10.3386/w27222

Cox, M., Arnold, G., \& Tomás, S. V. (2010). A review of design principles for community-based natural resource management. Ecology and Society, 15(4), Article 38.

Davidson, S., De Filippi, P., \& Potts, J. (2018). Blockchains and the economic institutions of capitalism. Journal of Institutional Economics, 14, 639-658.

De Filippi, P. (2018). Blockchain and the law: The rule of code. Harvard University Press.

De Filippi, P., \& Hassan, S. (2015). Measuring value in the commons-based ecosystem: Bridging the gap between the commons and the market. In G. Lovink \& N. Tkacz (Eds.), The moneylab reader (pp. 74-91). Institute of Network Cultures, University of Warwick.

De Filippi, P., \& Hassan, S. (2016). Blockchain technology as a regulatory technology: From code is law to law is code. First Monday, 21(12). https://doi.org/10.5210/fm.v21i12.7113

De Filippi, P., \& Loveluck, B. (2016). The invisible politics of Bitcoin: Governance crisis of a decentralised infrastructure. Internet Policy Review, 5(3). https://doi.org/10.14763/2016.3.427

De Filippi, P., \& Wright, A. (2018). Blockchain and the law. Blockchain and the Law: The Rule of Code.

Ducrée, J., Etzrodt, M., Gordijn, B., Gravitt, M., Bartling, S., Walshe, R., \& Harrington, T. (2020). Blockchain for organising effective grass-roots actions on a global commons: Saving the planet. Frontiers in Blockchain, 3, 1-33. https://doi. org/10.3389/fbloc. 2020.00033 
DuPont, Q. (2017). Experiments in algorithmic governance: A history and ethnography of "The DAO," a failed decentralized autonomous organization. In M. Campbell-Verduyn (Ed.), Bitcoin and beyond (pp. 157-177). Routledge.

El Faqir, Y., Arroyo, J., \& Hassan, S. (2020, August 26-27). An overview of decentralized autonomous organizations on the blockchain [Conference session]. 16th International Symposium on Open Collaboration (pp. 1-8), Madrid, Spain.

Eyal, I., \& Sirer, E. G. (2014). Majority is not enough: Bitcoin mining is vulnerable. In N. Christin \& R. Safavi-Naini (Eds.), Financial cryptography and data security (pp. 436-454). Springer. https://doi.org/10.1007/978-3-662-45472-5 28

Forte, A., Larco, V., \& Bruckman, A. (2009). Decentralization in Wikipedia governance. Journal of Management Information Systems, 26, 49-72. https://doi.org/10.2753/mis0742-1222260103

Franklin, S., \& Graesser, A. (1997). Is it an agent, or just a program? A taxonomy for autonomous agents. In J. P. Müller, M. J. Wooldridge, \& N. R. Jennings (Eds.), Intelligent agents III agent theories, architectures, and languages (pp. 21-35). Springer. https://doi.org/10.1007/bfb0013570

Freeman, J. (1972). The tyranny of structurelessness. Berkeley Journal of Sociology, 17, 151-164.

Frost, R. L., \& Postman, N. (1993). Technopoly: The surrender of culture to technology. Technology and Culture, 34, 714-715. https://doi.org/10.2307/3106743

Fuster-Morell, M. (2010). Governance of online creation communities for the building of digital commons. European University Institute. http://cadmus.eui.eu//handle/1814/14709

Fuster-Morell, M. (2014). Governance of online creation communities for the building of digital commons: Viewed through the framework of the institutional analysis and development. In K. Strandburg, B. Frischmann, K. Strandburg, \& M. Madison (Eds.), Governing knowledge commons (pp. 281-312). Oxford University Press.

Hardin, G. (1968). The tragedy of the commons. Science, 162(3859), 1243-1248.

Harris, C. (2018). Institutional solutions to free-riding in peer-topeer networks: A case study of online pirate communities. Journal of Institutional Economics, 14, 901-924. https://doi. org/10.1017/s1744137417000650

Hassan, S., Brekke, J. K., Atzori, M., Bodó, B., Meiklejohn, S., De Filippi, P., Beecroft, K., Rozas, D., Alonso, C. O., Vicente, E. M., Morales, G. L., \& Aguilar, A. F. (2020). Scanning the European ecosystem of distributed ledger technologies for social and public good: What, why, where, how, and ways to move forward [A. Pólvora, A. Hakami, \& E. Bol, Eds]. Publications Office of the European Union.

Hassan, S., \& De Filippi, P. (2021). Decentralized autonomous organizations (Glossary of distributed technologies). Internet Policy Review. https://policyreview.info/open-abstracts/decentralised-autonomous-organisation

Hayes, A. (2016). Decentralized banking: Monetary technocracy in the digital age. In P. Tasca, T. Aste, L. Pelizzon, \& N. Perony (Eds.), Banking beyond banks and money (pp. 121-131). Springer International.

Hess, C. (2008, July 14-18). Mapping the new commons. In proceedings of the 12th biennial conference of the international association for the study of the commons. Cheltenham, UK. SSRN Electronic Journal. https://doi.org/10.2139/ssrn. 1356835
Hess, C., \& Ostrom, E. (2007). Introduction: An overview of the knowledge commons. In C. Hess \& E. Ostrom (Eds.), Understanding Knowledge as a Commons: From Theory to Practice (pp. 3-26). MIT Press.

Heuermann, C. (2015). Governance 2.0: A Hayekian approach to (r)evolutionary self-governance by cryptocurrencies. University of Konstanz.

Huckle, S., \& White, M. (2016). Socialism and the blockchain. Future Internet, 8(4), Article 49.

Hutchby, I. (2001). Technologies, texts and affordances. Sociology, 35, 441-456. https://doi.org/10.1017/s0038038501000219

Jemielniak, D. (2016). Wikimedia movement governance: The limits of a-hierarchical organization. Journal of Organizational Change Management, 29, 361-378. https://doi.org/10.1108/ jocm-07-2013-0138

Johnston, D. A. (2014, August 30). Everything will be decentralized. Medium. https://medium.com/@DJohnstonEC/everything-willbe-decentralized-d7dcedca45e

Juris, J. S. (2016). Reflections on \#occupy everywhere: Social media, public space, and emerging logics of aggregation. In $\mathrm{C}$. Feixa, C. Leccardi, \& P. Nilan (Eds.), Youth, space and time (pp. 385-414). Brill. https://doi.org/10.1163/9789004324589_021

Kabbinale, A. R., Dimogerontakis, E., Selimi, M., Ali, A., Navarro, L., Sathiaseelan, A., \& Crowcroft, J. (2019). Blockchain for economically sustainable wireless mesh networks. Concurrency and Computation: Practice and Experience, 32, Article e5349. https://doi.org/10.1002/cpe.5349

Khan, J. (2017). To what extent can blockchain be used as a tool for community guidance. http://heinonline.org/ HOL/Page?handle=hein.journals/edinslr3\&div $=33 \& \mathrm{~g}$ sent $=1 \&$ casa_token $=\&$ collection $=$ journals $\#$

Koehler, W. (1999). Digital libraries and world wide web sites and page persistence. Information Research, 4(4). http://informationr.net/ir/4-4/paper60.html

Le Breton, D. (2015). Disparaitre de soi: Une tentation contemporaine [Dissappearing from oneself: A contemporary temptation]. Anne-Marie Métailié.

Liu, P. T. S. (2016). Medical record system using blockchain, big data and tokenization. In K. Y. Lam, C. H. Chi, \& S. Qing (Eds.), Information and communications security (pp. 254 261). Springer. https://doi.org/10.1007/978-3-319-50011-9_20

Lo, Y. C., \& Medda, F. (2020). Assets on the blockchain: An empirical study of tokenomics. Information Economics and Policy, 53, Article 100881.

Marx, K. (1875). Critique of the Gotha programme. Part I. https:// www.marxists.org/archive/marx/works/1875/gotha/ch01.htm

Mateos-García, J., \& Steinmueller, W. E. (2008). The institutions of open source software: Examining the Debian community. Information Economics and Policy, 20, 333-344. https://doi. org/10.1016/j.infoecopol.2008.06.001

Mayer-Schonberger, V. (2009). Delete: The virtue of forgetting in the digital age. Princeton University Press.

Nakamoto, S. (2008). Bitcoin: A peer-to-peer electronic cash system. http://bitcoin.org/bitcoin.pdf

Nguyen, Q. K. (2016). Blockchain-A financial technology for future sustainable development. IEEE Conference Publication. https://doi.org/10.1109/GTSD.2016.22

O'Dwyer, R. (2015, November 6). The revolution will (not) Be decentralized: Blockchains. http://commonstransition.org/therevolution-will-not-be-decentralised-blockchains/ 
Ølnes, S., Ubacht, J., \& Janssen, M. (2017). Blockchain in government: Benefits and implications of distributed ledger technology for information sharing. Government Information Quarterly, 34, 355-364. https://doi.org/10.1016/j.giq.2017.09.007

Ostrom, E. (1990). Governing the commons: The evolution of institutions for collective action. Cambridge University Press.

Ostrom, E. (2000). Collective action and the evolution of social norms. The Journal of Economic Perspectives: A Journal of the American Economic Association, 14(3), 137-158.

Ostrom, E. (2009). Understanding institutional diversity. Princeton University Press. https://doi.org/10.2307/j.ctt7s7wm

Pazaitis, A., De Filippi, P., \& Kostakis, V. (2017). Blockchain and value systems in the sharing economy: The illustrative case of backfeed. Technological Forecasting and Social Change, 125, 105-115.

Pérez-Orozco, A. (2014). Subversión Feminista de La Economía. Aportes Para Un Debate Sobre El Conflicto Capital-Vida [A feminist subversion of the economy. Contributions for a debate on the capital-life conflict]. Traficantes de sueños.

Pitt, J., Diaconescu, A., \& Bollier, D. (2017, June 29). Technology for collective action. IEEE Technology and Society. https:// technologyandsociety.org/technology-for-collective-action/

Pitt, J., Schaumeier, J., \& Artikis, A. (2012). Axiomatization of socio-economic principles for self-organizing institutions: Concepts, experiments and challenges. ACM Transactions on Autonomous and Adaptive Systems, 7(4), 1-39.

Platero, R. (2014). ¿Es El Análisis Interseccional Una Metodología Feminista Y Queer? [Is intersectional analysis a feminist and queer methodology?] In Reflexiones, Herramientas $Y$ Aplicaciones Desde La Investigación Feminista [Reflections, tools and applications from feminist research] (pp. 79-95). Instituto Hegoa.

Reber, D., \& Feuerstein, S. (2014). Bitcoins-hype or real alternative (IFI-2014.01). University of Zürich.

Reyna, A., Martín, C., Chen, J., Soler, E., \& Díaz, M. (2018). On blockchain and its integration with IoT. Challenges and opportunities. Future Generations Computer Systems: FGCS, 88, 173-190.

Risius, M., \& Spohrer, K. (2017). A blockchain research framework: What we (don't) know, where we go from here, and how we will get there. Business \& Information Systems Engineering, 59(6), 385-409.

Rosen, J. (2010, July). The web means the end of forgetting. The New York Times. https://www.nytimes.com/2010/07/25/magazine/ 25 privacy-t $2 . \mathrm{html}$.

Rozas, D. (2017). Self-organisation in commons-based peer production: Drupal: "The drop is always moving" [PhD thesis]. University of Surrey. http://epubs.surrey.ac.uk/845121/

Rozas, D. (2020). Affordances of decentralised technologies for commons-based governance of shared technical infrastructure. Prospectives, 1, 1, Bartlett School of Architecture, University College of London. ISSN 2634-8578.

Rozas, D., \& Díaz-Molina, S. (2019, June 24-27). Potentialities and limitations of blockchain technologies in the governance of social enterprise collectives: The case of smart Ibérica [Conference session]. 7th EMES International Research Conference. Sheffield, UK. https://doi.org/10.2139/ ssrn.3346561

Rozas, D., Gilbert, N., Hodkinson, P., \& Hassan, S. (2021). Talk is silver, code is gold? Beyond traditional notions of contribution in peer production: The case of Drupal. Frontiers in Human
Dynamics. https://www.frontiersin.org/articles/10.3389/fhumd. 2021.618207/

Rozas, D., \& Huckle, S. (2021). Loosen control without losing control: Formalization and decentralization within commons-based peer production. Journal of the Association for Information Science and Technology, 72, 204-223. https://doi.org/10.1002/ asi.24393

Salcedo, J. L., \& Fuster-Morell, M. (2014, September 25-26). Mapping the common based peer production: A crowd-sourcing experiment [Conference session]. The Internet, Policy \& Politics Conferences. Oxford, UK. http://blogs.oii.ox.ac.uk/ ipp-conference/2014/programme-2014/track-a-harnessing-thecrowd/design-i/mayo-fuster-morell-ruben-mart $\% \mathrm{C} 3 \% \mathrm{ADnez-}$ jorge.html

Schweik, C. M., \& English, R. (2013). Preliminary steps toward a general theory of internet-based collective-action in digital information commons: Findings from a study of open source software projects. International Journal of the Commons, 7(2), 234-254.

Shackelford, S., \& Myers, S. (2017). Block-by-block: Leveraging the power of blockchain technology to build trust and promote cyber peace. Yale Journal of Law \& Technology, 19, 334. https://heinonline.org/HOL/LandingPage?handle=hein.journals/yjolt19\&div $=8 \&$ id $=\&$ page $=$

Sharon, T., \& Zandbergen, D. (2017). From data fetishism to quantifying selves: Self-tracking practices and the other values of data. New Media \& Society, 19(11), 1695-1709.

Shaw, A., \& Hill, B. (2014). Laboratories of oligarchy? How the iron law extends to peer production. Journal of Communication, 64(2), 215-238.

Sullivan, L. E. (2009). The SAGE glossary of the social and behavioral sciences. Sage.

Swan, M. (2015). Blockchain: Blueprint for a new economy. O'Reilly.

Szabo, N. (1997). Formalizing and securing relationships on public networks. First Monday. https://doi.org/10.5210/fm.v2i9.548

Thierer, A. (2016). Permissionless innovation: The continuing case for comprehensive technological freedom. Mercatus Center at George Mason University.

Tkacz, N. (2014). Wikipedia and the politics of openness. University of Chicago Press. https://doi.org/10.7208/chicago/9780226192444.001.0001

Underwood, S. (2016). Blockchain beyond bitcoin. Communications of the ACM, 59(11), 15-17. https://doi.org/10.1145/ 2994581

Viégas, F. B., Wattenberg, M., \& McKeon, M. M. (2007). The hidden order of Wikipedia. In D. Schuler (Ed.), Online communities and social computing (pp. 445-454). Springer.

Wellman, B. (2004). The Glocal village: Internet and community. The Arts \& Science Review, 1, 26-29.

Wellman, B., Quan-Haase, A., Boase, J., Chen, W., Hampton, K., Díaz, I., \& Miyata, K. (2006). The social affordances of the internet for networked individualism. Journal of ComputerMediated Communication, 8(3), JCMC834. https://doi.org/10. 1111/j.1083-6101.2003.tb00216.x

Werbach, K. (2018). The blockchain and the new architecture of trust. The MIT Press. https://doi.org/10.7551/mitpress/11449.001.0001

Wright, A., \& De Filippi, P. (2015). Decentralized blockchain technology and the rise of lex cryptographia. https://papers.ssrn. com/sol3/papers.cfm?abstract_id=2580664 